Proyecciones Journal of Mathematics

Vol. 30, No 1, pp. 29-41, May 2011.

Universidad Católica del Norte

Antofagasta - Chile

DOI: 10.4067/S0716-09172011000100003

\title{
On strongly faint e-continuous functions
}

\author{
Miguel Caldas \\ Universidade Federal Fluminense, Brasil \\ and \\ Saeid Jafari \\ College of Vestsjaelland South, Denmark \\ Received: June 2010. Accepted : March 2011
}

\begin{abstract}
A new class of functions, called strongly faint e-continuous function, has been defined and studied.

Relationships among strongly faint $e$-continuous functions and $e$ connected spaces, e-normal spaces and e-compact spaces are investigated. Furthermore, the relationships between strongly faint e-continuous functions and graphs are also investigated.
\end{abstract}

2000 Mathematics Subject Classification : 54B05, 54C08, $54 D 10$.

Key words and phrases : Topological spaces, e-open sets, strong $\theta$-continuity, strongly faint e-continuity. 


\section{Introduction}

Recent progress in the study of characterizations and generalizations of continuity, compactness, connectedness, separation axioms etc. has been done by means of several generalized closed sets. The first step of generalizing closed set was done by Levine in 1970 [13]. The notion of generalized closed sets has been studied extensively in recent years by many topologists. As a generalization of closed sets, $e$-closed sets and the related sets were introduced and studied by E. Ekici ([4], [5], [6], [7], [8], [9]).

Nasef and Noiri [18] introduce three classes of strong forms of faintly continuity namely: strongly faint semicontinuity, strongly faint precontinuity and strongly faint $\beta$-continuity. Recently Nasef [16] defined strong forms of faint continuity under the terminologies strongly faint $\alpha$-continuity and strongly faint $\gamma$-continuity. In this paper using $e$-open sets, strongly faint $e$-continuity is introduced and studied. Moreover, basic properties and preservation theorems of strongly faint $e$ - continuous functions are investigated and relationships between strongly faint $e$-continuous functions and graphs are investigated.

\section{Preliminaries}

Throughout the paper $(X, \tau)$ and $(Y, \sigma)$ (or simply $X$ and $Y$ ) represent topological spaces on which no separation axioms are assumed unless otherwise mentioned. For a subset $A$ of a space $(X, \tau), \operatorname{cl}(A), \operatorname{int}(A)$ and $X \backslash A$ denote the closure of $A$, the interior of $A$ and the complement of $A$ in $X$, respectively. A point $x \in X$ is called a $\theta$-cluster [23] (resp. $\delta$-cluster [23]) point of $A$ if $\operatorname{cl}(V) \cap A \neq \emptyset$ (resp. $\operatorname{int}(\operatorname{cl}(V)) \cap A \neq \emptyset$ ) for every open set $V$ of $X$ containing $x$. The set of all $\theta$-cluster (resp. $\delta$-cluster) points of $A$ is called the $\theta$-closure (resp. $\delta$-closure) of $A$ and is denoted by $c l_{\theta}(A)$ (resp. $\delta c l(A)$ ). If $A=\operatorname{cl}_{\theta}(A)$ (resp. $A=\delta c l(A)$ ), then $A$ is said to be $\theta$-closed (resp. $\delta$-closed). The complement of a $\theta$-closed (resp. $\delta$-closed) set is said to be $\theta$-open [23](resp. $\delta$-open [23]). The union of all $\theta$-open sets contained in a subset $A$ is called the $\theta$-interior of $A$ and is denoted by $\operatorname{int}_{\theta}(A)$. It follows from [23] that the collection of $\theta$-open sets in a topological space $(X, \tau)$ forms a topology $\tau_{\theta}$ on $X[14]$. The family of all $\theta$-open (resp. $\theta$-closed) subsets of $X$ is denoted by $\theta O(X)$ (resp. $\theta C(X)$ ).

We recall the following definitions, which are well-known and are useful in the sequel. 
Definition 1. A subset $A$ of a space $(X, \tau)$ is called semi-open [12] (resp. $\alpha$-open [19], preopen [15], $\beta$-open [1], $\gamma$-open [10], $\delta$-preopen [21]) if $A \subset$ $\operatorname{cl}(\operatorname{Int}(A))(\operatorname{resp} . A \subset \operatorname{int}(\operatorname{cl}(\operatorname{int}(A))), A \subset \operatorname{int}(\operatorname{cl}(A)), A \subset \operatorname{cl}(\operatorname{int}(\operatorname{cl}(A)))$, $A \subset \operatorname{cl}(\operatorname{int}(A)) \cup \operatorname{int}(\operatorname{cl}(A)), A \subset \operatorname{int}(\delta c l(A)))$.

A subset $A$ of a topological space $X$ is said to be $e$-open [4] if $A \subset$ $\operatorname{int}(\delta c l(A)) \cup \operatorname{cl}(\operatorname{sint}(A))$. The complement of an $e$-open set is said to be $e$ closed [4]. The intersection (union) of all $e$-closed ( $e$-open) sets containing (contained in) $A$ in $X$ is called the $e$-closure [4] (the $e$-interior [4]) of $A$ and is denoted by $e-c l(A)$ (resp. e-int $(A)$ ). By $e O(X)$ or $e O(X, \tau)$ (resp. $e C(X))$, we denote the collection of all $e$-open (resp. $e$-closed) sets of $X$. We set $e O(X, x)=\{U: x \in U \in e O(X)\}$. Similarly, we denote the collection of all $\gamma$-open, semiopen, preopen and $\beta$-open sets by $\gamma O(X, \tau), S O(X, \tau)$, $P O(X, \tau)$ and $\beta O(X, \tau)$, respectively.

Lemma 2.1. ([4], [5], [6], [8]). The following properties holds for the eclosure of a set in a space $X$ :

(1) Arbitrary union (intersection) of e-open (e-closed) sets in $X$ is e-open (resp. e-closed).

(2) $A$ is e-closed in $X$ if and only if $A=e-c l(A)$.

(3) $e-c l(A) \subset e-c l(B)$ whenever $A \subset B(\subset X)$.

(4) $e-c l(A)$ is e-closed in $X$.

(5) $e-\operatorname{cl}(e-c l(A))=e-c l(A)$.

(6) $e$-cl $(A)=\{x \in X \mid U \cap A \neq \emptyset$ for every e-open set $U$ containing $x\}$.

We have the following diagram in which the converses of implications need not be true, as is showed in [4].

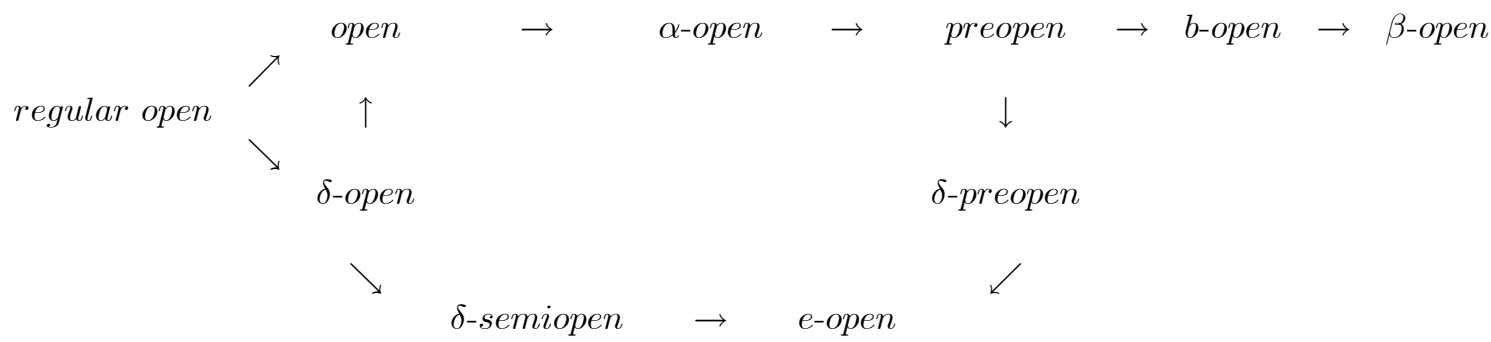

Definition 2. A function $f:(X, \tau) \rightarrow(Y, \sigma)$ is said to be strongly faint semicontinuous [18] (resp. strongly faint precontinuous [17], strongly faint $\beta$-continuous [18], strongly faint $\alpha$-continuous [16], strongly faint $\gamma$-continuous 
[16]) if for each $x \in X$ and each semiopen (resp. preopen, $\beta$-open, $\alpha$-open, $\gamma$-open) set $V$ of $Y$ containing $f(x)$, there exists a $\theta$-open set $U$ of $X$ containing $x$ such that $f(U) \subset V$.

Definition 3. A function $f:(X, \tau) \rightarrow(Y, \sigma)$ is said to be:

(i) quasi $\theta$-continuous [11], if $f^{-1}(V)$ is $\theta$-open in $X$ for every $\theta$-open set $V$ of $Y$.

(ii) e-irresolute [6, 8], if $f^{-1}(V)$ is e-open in $X$ for every e-open set $V$ of $Y$.

(iii) e-continuous [4], if $f^{-1}(V)$ is e-open in $X$ for every open set $V$ of $Y$.

(iv) strongly $\theta$-continuous [20], if $f^{-1}(V)$ is $\theta$-open in $X$ for every open set $V$ of $Y$.

\section{3. strongly faint $e$-continuous functions}

Definition 4. A function $f:(X, \tau) \rightarrow(Y, \sigma)$ is said to be

(i) strongly faint e-continuous if for each $x \in X$ and each e-open set $V$ of $Y$ containing $f(x)$, there exists a $\theta$-open set $U$ of $X$ containing $x$ such that $f(U) \subset V$.

(ii) strongly e-continuous if $f^{-1}(V)$ is open in $X$ for every e-open set $V$ of $Y$.

Theorem 3.1. For a function $f:(X, \tau) \rightarrow(Y, \sigma)$, the following statements are equivalent:

(i) $f$ is strongly faint e-continuous;

(ii) $f:\left(X, \tau_{\theta}\right) \rightarrow(Y, \sigma)$ is strongly e-continuous;

(iii) $f^{-1}(V)$ is $\theta$-open in $X$ for every e-open set $V$ of $Y$;

(iv) $f^{-1}(F)$ is $\theta$-closed in $X$ for every e-closed subset $F$ of $Y$.

Proof. (i) $\Rightarrow$ (iii): Let $V$ be an $e$-open set of $Y$ and $x \in f^{-1}(V)$. Since $f(x) \in V$ and $f$ is strongly faint $e$-continuous, there exists a $\theta$-open set $U$ of $X$ containing $x$ such that $f(U) \subset V$. It follows that $x \in U \subset f^{-1}(V)$. Hence $f^{-1}(V)$ is $\theta$-open in $X$.

(iii) $\Rightarrow($ i): Let $x \in X$ and $V$ be an $e$-open set of $Y$ containing $f(x)$. By (iii), $f^{-1}(V)$ is a $\theta$-open set containing $x$. Take $U=f^{-1}(V)$. Then $f(U) \subset V$. This shows that $f$ is strongly faint $e$-continuous.

(iii) $\Rightarrow$ (iv): Let $V$ be any $e$-closed set of $Y$. Since $Y \backslash V$ is an $e$-open set, by (iii), it follows that $f^{-1}(Y \backslash V)=X \backslash f^{-1}(V)$ is $\theta$-open. This shows that $f^{-1}(V)$ is $\theta$-closed in $X$.

(iv) $\Rightarrow$ (iii): Let $V$ be an $e$-open set of $Y$. Then $Y \backslash V$ is $e$-closed in $Y$. By (iv), $f^{-1}(Y \backslash V)=X \backslash f^{-1}(V)$ is $\theta$-closed and thus $f^{-1}(V)$ is $\theta$-open. 
(i) $\Leftrightarrow($ ii): Clear.

The relationships between this new class of functions and other corresponding types of functions are shown in the following diagram.

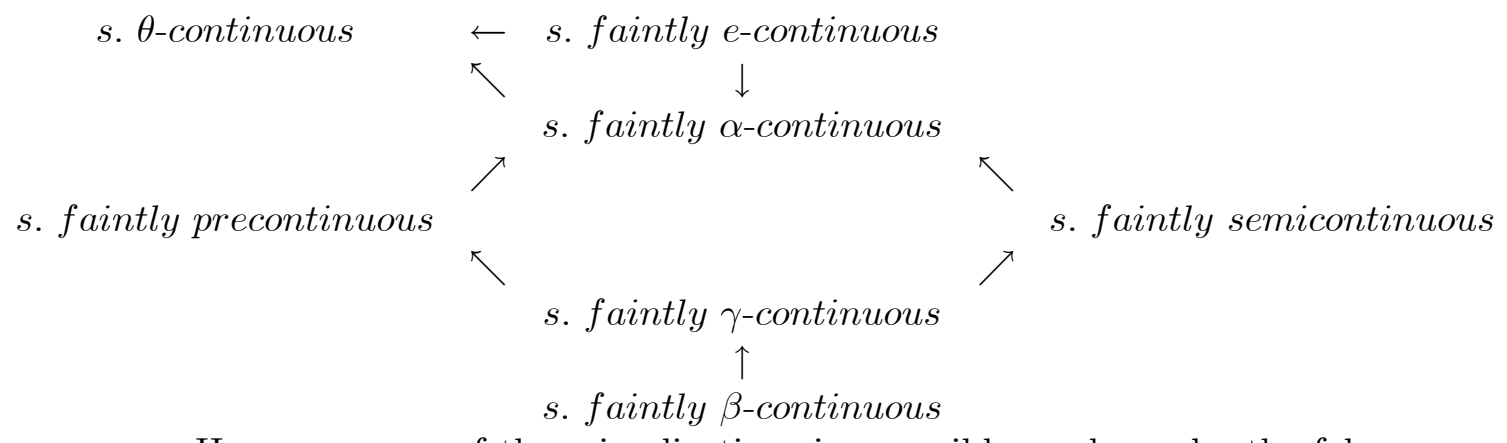

However, none of these implications is reversible as shown by the following examples and well-known facts.

Example 3.2. (i) In ([18], Examples 3.2), it is shown that a strong faint semicontinuity which is not a strong faint precontinuity.

(ii) In ([16], Examples 4.3, (resp. Examples: 4.4 and 4.5)), it is shown a strong faint semicontinuity which is not a strong faint $\gamma$-continuity (resp. a strong faint precontinuity which is not a strong faint $\gamma$-continuity and a strong faint $\gamma$-continuity which is not a strong faint $\beta$-continuity).

(iii) Using Example 3.2 of [18], this is easily observed that a strongly faint $\alpha$-continuity need not be strongly faint e-continuity.

Theorem 3.3. If a function $f:(X, \tau) \rightarrow(Y, \sigma)$ is strongly faint e-continuous function, then it is strongly e-continuous.

If $(X, \tau)$ is a regular space, we have $\tau=\tau_{\theta}$ and the next theorem follows immediately.

Theorem 3.4. Let $(X, \tau)$ be a regular space. Then for a function $f$ : $(X, \tau) \rightarrow(Y, \sigma)$ the following properties are equivalent:

(i) $f$ is strongly e-continuous.

(ii) $f$ is strongly faint e-continuous.

Recall that, a topological space $(Y, \sigma)$ is said to be a $T_{e}$-space [2] if every $e$-open subset of $(Y, \sigma)$ is open. 
A space $X$ is said to be submaximal if each dense subset of $X$ is open in $X$ and extremaly disconnected (briefly ED) if the closure of each open set of $X$ is open in $X$.

Theorem 3.5. Let $(Y, \sigma)$ be a submaximal $E D, T_{e}$-space. Then the following are equivalent for a function $f:(X, \tau) \rightarrow(Y, \sigma)$ :

(i) $f$ is strongly faint $\alpha$-continuous,

(ii) $f$ is strongly faint $\gamma$-continuous,

(iii) $f$ is strongly faint semicontinuous.

(iv) $f$ is strongly faint precontinuous.

(v) $f$ is strongly faint $\beta$-continuous.

(vi) $f$ is strongly $\theta$-continuous.

(vii) $f$ is strongly faint e-continuous.

Proof. From [18], we have $(i) \Rightarrow(i i) \Rightarrow(i i i) \Rightarrow(i v) \Rightarrow(v) \Rightarrow(v i) \Rightarrow(i)$ (since $X$ is submaximal and $D E$, then $\sigma=\sigma^{\alpha}=\gamma O(Y, \sigma)=S O(Y, \sigma)=$ $P O(Y, \sigma)=\beta O(Y, \sigma))$.

$(v i) \Leftrightarrow(v i i)$ : This follows from the fact that if $(Y, \sigma)$ is $T_{e}$-space, then $\sigma=e O(Y, \sigma)$.

Theorem 3.6. If $f: X \rightarrow Y$ is strongly faint e-continuous and $g: Y \rightarrow Z$ is e-irresolute, then $g \circ f: X \rightarrow Z$ is strongly faint $e$-continuous.

Proof. Let $G \in e O(Z)$. Then $g^{-1}(G) \in e O(Y)$ and hence $(g \circ f)^{-1}(G)=$ $f^{-1}\left(g^{-1}(G)\right)$ is $\theta$-open in $X$. Therefore $g \circ f$ is strongly faint $e$-continuous.

Theorem 3.7. The following statements hold for functions $f: X \rightarrow Y$ and $g: Y \rightarrow Z$ :

(i) If both $f$ and $g$ are strongly faint e-continuous, then the composition $g \circ f: X \rightarrow Z$ is strongly faint e-continuous.

(ii) If $f$ strongly faint $e$-continuous and $g$ is an e-irresolute, then $g \circ f$ is strongly e-continuous.

(iii) If $f$ strongly faint $e$-continuous and $g$ is a $e$-continuous, then $g \circ f$ is strongly $\theta$-continuous.

(iv) If $f$ is quasi $\theta$-continuous and $g$ is strongly faint $e$-continuous, then $g \circ f$ is strongly faint $e$-continuous.

(v) If $f$ is strongly $\theta$-continuous and $g$ is strongly faint $e$-continuous, then $g \circ f$ is strongly faint e-continuous.

Definition 5. A $\theta$-frontier [11] of a subset $A$ of $(X, \tau)$ is $\theta F r(A)=\operatorname{cl}_{\theta}(A) \cap$ $c l_{\theta}(X \backslash A)$. 
Theorem 3.8. Let $(X, \tau)$ be a regular space. Then the set of all points $x \in X$ in which a function $f:(X, \tau) \rightarrow(Y, \sigma)$ is not strongly faint $e$ continuous at $x$ is identical with the union of the $\theta$-frontier of the inverse images of e-open subsets of $Y$ containing $f(x)$.

Proof. Necessity. Suppose that $f$ is not strongly faint $e$-continuous at $x \in X$. Then there exists a $e$-open set $V$ of $Y$ containing $f(x)$ such that $f(U)$ is not a subset of $V$ for each $U \in \tau_{\theta}$ containing $x$. Hence we have $U \cap\left(X \backslash f^{-1}(V)\right) \neq \emptyset$ for each $U \in \tau_{\theta}$ containing $x$. Since $X$ is regular, it follows that $x \in \operatorname{cl}_{\theta}\left(X \backslash f^{-1}(V)\right)$. On the other hand we have that, $x \in f^{-1}(V) \subset \operatorname{cl}_{\theta}\left(f^{-1}(V)\right)$. This means that $x \in \theta F r\left(f^{-1}(V)\right)$.

Sufficiency. Suppose that $x \in \theta F r\left(f^{-1}(V)\right)$ for some $V \in e O(Y, f(x))$. Now, we assume that $f$ is strongly faint $e$-continuous at $x \in X$. Then there exists $U \in \tau_{\theta}$ containing $x$ such that $f(U) \subset V$. Therefore, we have $U \subset f^{-1}(V)$ and hence $x \in \operatorname{int}_{\theta}\left(f^{-1}(V)\right) \subset X \backslash \theta F r\left(f^{-1}(V)\right)$. This is a contradiction. This means that $f$ is not strongly faint $e$-continuous.

Definition 6. (i) A space $(X, \tau)$ is said to be e-connected [6, 8] (resp. $\theta$-connected [11]) if $X$ cannot be written as the union of two nonempty disjoint e-open (resp. $\theta$-open) sets.

(ii) A subset $K$ of a $(X, \tau)$ space is said to be, e-compact [6, 8] (resp. $\theta$ compact [11]) relative to $(X, \tau)$, if for every cover of $K$ by e-open (resp. $\theta$-open) sets has a finite subcover. A topological space $(X, \tau)$ is e-compact $[6,8]$ (resp. $\theta$-compact) if the set $X$ is e-compact (resp. $\theta$-compact) relative to $(X, \tau)$.

It should be mentioned that $\theta$-connected is equivalent with connected (see [11]).

Theorem 3.9. If $f:(X, \tau) \rightarrow(Y, \sigma)$ is a strongly faint e-continuous surjection function and $(X, \tau)$ is a $\theta$-connected space, then $Y$ is an $e$-connected space.

Proof. Assume that $(Y, \sigma)$ is not $e$-connected. Then there exist nonempty $e$-open sets $V_{1}$ and $V_{2}$ of $Y$ such that $V_{1} \cap V_{2}=\emptyset$ and $V_{1} \cup V_{2}=Y$. Hence we have $f^{-1}\left(V_{1}\right) \cap f^{-1}\left(V_{2}\right)=\emptyset$ and $f^{-1}\left(V_{1}\right) \cup f^{-1}\left(V_{2}\right)=X$. Since $f$ is surjective, $f^{-1}\left(V_{1}\right)$ and $f^{-1}\left(V_{2}\right)$ are nonempty subsets of $X$. Since $f$ is strongly faint $e$-continuous, $f^{-1}\left(V_{1}\right)$ and $f^{-1}\left(V_{2}\right)$ are $\theta$-open sets of $X$. Therefore $(X, \tau)$ is not $\theta$-connected. This is a contradiction and hence $(Y, \sigma)$ is $e$-connected. 
Theorem 3.10. If $f:(X, \tau) \rightarrow(Y, \sigma)$ is a strongly faint e-continuous, then $f(K)$ is e-compact relative to $(Y, \sigma)$ for each subset $K$ which is $\theta$ compact relative to $(X, \tau)$.

Proof. Let $\left\{V_{i}: i \in I\right\}$ be any cover of cover of $f(K)$ by $e$-open sets. For each $x \in K$, there exists $i_{x} \in I$, such that $f(x) \in V_{i_{x}}$. Since $f$ is strongly faint $e$-continuous, there exists $U_{x} \in \tau_{\theta}$ containing $x$ such that $f\left(U_{x}\right) \subset V_{i_{x}}$. The family $\left.\left\{U_{x}\right): x \in K\right\}$ is a cover of $K$ by $\theta$-open sets of $(X, \tau)$. Since $K$ is $\theta$-compact relative to $(X, \tau)$, there exists a finite subset $K_{0}$ of $K$ such that $K \subset \bigcup\left\{U_{x}: x \in K_{0}\right\}$. Therefore, we obtain $f(K) \subset \bigcup\left\{f\left(U_{x}\right): x \in K_{0}\right\} \subset \bigcup\left\{V_{i_{x}}: x \in K_{0}\right\}$. Therefore, $f(K)$ is $e$-compact relative to $(Y, \sigma)$.

Theorem 3.11. The surjective strongly faint e-continuous image of a $\theta$ compact space is e-compact.

Proof. Let $f:(X, \tau) \rightarrow(Y, \sigma)$ be a strongly faint $e$-continuous function from a $\theta$-compact space $X$ onto a space $Y$. Let $\left\{G_{\alpha}: \alpha \in I\right\}$ be any $e$-open cover of $Y$. Since $f$ is strongly faint $e$-continuous, $\left\{f^{-1}\left(G_{\alpha}\right): \alpha \in I\right\}$ is a $\theta$-open cover of $X$. Since $X$ is $\theta$-compact, there exists a finite subcover $\left\{f^{-1}\left(G_{i}\right): i=1,2, \ldots n\right\}$ of $X$. Then it follows that $\left\{G_{i}: i=1,2, \ldots n\right\}$ is a finite subfamily which cover $Y$. Hence $Y$ is $e$-compact.

\section{Separation Axioms}

Recall that a topological space $(X, \tau)$ is said to be:

(i) $e-T_{1}[6,8]$ (resp. $\theta-T_{1}$ ) if for each pair of distinct points $x$ and $y$ of $X$, there exists $e$-open (resp. $\theta$-open) sets $U$ and $V$ containing $x$ and $y$, respectively such that $y \notin U$ and $x \notin V$.

(ii) $e-T_{2}[6,8]$ (resp. $\theta-T_{2}$ [22]) if for each pair of distinct points $x$ and $y$ in $X$, there exists disjoint $e$-eopen (resp. $\theta$-open) sets $U$ and $V$ in $X$ such that $x \in U$ and $y \in V$.

Remark 4.1. [3] Hausdorff $\Leftrightarrow \theta-T_{1}$.

Theorem 4.2. If $f:(X, \tau) \rightarrow(Y, \sigma)$ is strongly faint e-continuous injection and $Y$ is an $e-T_{1}$ space, then $X$ is a $\theta-T_{1}$ (or Hausdorff) space.

Proof. Suppose that $Y$ is $e-T_{1}$. For any distinct points $x$ and $y$ in $X$, then there exist $V, W \in e O(Y)$ such that $f(x) \in V, f(y) \notin V, f(x) \notin W$ 
and $f(y) \in W$. Since $f$ is strongly faint $e$-continuous, $f^{-1}(V)$ and $f^{-1}(W)$ are $\theta$-open subsets of $(X, \tau)$ such that $x \in f^{-1}(V), y \notin f^{-1}(V), x \notin f^{-1}(W)$ and $y \in f^{-1}(W)$. This shows that $X$ is $\theta-T_{1}$ (equivalently Hausdorff by Remark 4.1).

Theorem 4.3. If $f:(X, \tau) \rightarrow(Y, \sigma)$ is strongly faint e-continuous injection and $Y$ is an $e-T_{2}$ space, then $X$ is a $\theta-T_{2}$ space.

Proof. Suppose that $Y$ is $e-T_{2}$. For any pair of distinct points $x$ and $y$ in $X$, there exist disjoint $e$-open sets $U$ and $V$ in $Y$ such that $f(x) \in U$ and $f(y) \in V$. Since $f$ is strongly faint $e$-continuous, $f^{-1}(U)$ and $f^{-1}(V)$ are $\theta$-open in $X$ containing $x$ and $y$, respectively. Therefore, $f^{-1}(U) \cap f^{-1}(V)$ $=\emptyset$ because $U \cap V=\emptyset$. This shows that $X$ is $\theta-T_{2}$.

Theorem 4.4. If $f, g: X \rightarrow Y$ are strongly faint $e$-continuous functions and $Y$ is $e-T_{2}$, then $E=\{x \in X: f(x)=g(x)\}$ is closed in $X$.

Proof. Suppose that $x \notin E$. Then $f(x) \neq g(x)$. Since $Y$ is $e-T_{2}$, there exist $V \in e O(Y, f(x))$ and $W \in e O(Y, g(x))$ such that $V \cap W=\emptyset$. Since $f$ and $g$ are strongly faint $e$-continuous, there exist a $\theta$-open set $U$ of $X$ containing $x$ and a $\theta$-open set $G$ of X containing $x$ such that $f(U) \subset V$ and $g(G) \subset W$. Set $D=U \cap G$. then $D \cap E=\emptyset$ with $D$ a $\theta$-open subset and hence open such that $x \in D$. Then $x \notin \operatorname{cl}(E)$ and thus $E$ is closed in $X$.

Definition 7. A space $(X, \tau)$ is said to be: (i) $\theta$-regular (resp. e-regular [5]) if for each $\theta$-closed (resp. e-closed) set $F$ and each point $x \notin F$, there exist disjoint $\theta$-open (resp. e-open) sets $U$ and $V$ such that $F \subset U$ and $x \in V$.

(ii) $\theta$-normal (resp. e-normal [5]) if for any pair of disjoint $\theta$-closed (resp. $e$-closed) subsets $F_{1}$ and $F_{2}$ of $X$, there exist disjoint $\theta$-open (resp. e-open) sets $U$ and $V$ such that $F_{1} \subset U$ and $F_{2} \subset V$.

Recall that a function $f:(X, \tau) \rightarrow(Y, \sigma)$ is called $\theta e$-open if $f(V) \in$ $e O(Y)$ for each $V \in \tau_{\theta}$.

Theorem 4.5. If $f$ is strongly faint $e$-continuous $\theta e$-open injection from a $\theta$-regular space $(X, \tau)$ onto a space $(Y, \sigma)$, then $(Y, \sigma)$ is e-regular.

Proof. Let $F$ be an $e$-closed subset of $Y$ and $y \notin F$. Take $y=f(x)$. Since $f$ is strongly faint $e$-continuous, $f^{-1}(F)$ is $\theta$-closed in $X$ such that $f^{-1}(y)=x \notin f^{-1}(F)$. Take $G=f^{-1}(F)$. We have $x \notin G$. Since $X$ is 
$\theta$-regular, then there exist disjoint $\theta$-open sets $U$ and $V$ in $X$ such that $G \subset U$ and $x \in V$. We obtain that $F=f(G) \subset f(U)$ and $y=f(x) \in f(U)$ such that $f(U)$ and $f(V)$ are disjoint $e$-open sets. This shows that $Y$ is $e$-regular.

Theorem 4.6. If $f$ is strongly faint $e$-continuous $\theta e$-open injection from a $\theta$-normal space $(X, \tau)$ onto a space $(Y, \sigma)$, then $Y$ is e-normal.

Proof. Let $F_{1}$ and $F_{2}$ be disjoint $e$-closed subsets of $Y$. Since $f$ is strongly faint $e$-continuous, $f^{-1}\left(F_{1}\right)$ and $f^{-1}\left(F_{2}\right)$ are $\theta$-closed sets. Take $U$ $=f^{-1}\left(F_{1}\right)$ and $V=f^{-1}\left(F_{2}\right)$. We have $U \cap V=\emptyset$. Since $X$ is $\theta$-normal, there exist disjoint $\theta$-open sets $A$ and $B$ such that $U \subset A$ and $V \subset B$. We obtain that $F_{1}=f(U) \subset f(A)$ and $F_{2}=f(V) \subset f(B)$ such that $f(A)$ and $f(B)$ are disjoint $e$-open sets. Thus, $Y$ is $e$-normal.

Recall that for a function $f:(X, \tau) \rightarrow(Y, \sigma)$, the subset $\{(x, f(x))$ : $x \in X\} \subset X \times Y$ is called the graph of $f$ and is denoted by $G(f)$.

Definition 8. A graph $G(f)$ of a function $f:(X, \tau) \rightarrow(Y, \sigma)$ is said to be $(e, \theta)$-closed if for each $(x, y) \in(X \times Y) \backslash G(f)$, there exist a $\theta$-open $U$ set of $X$ containing $x$ and an e-open set $V$ of $Y$ containing $y$ such that $(U \times V)$ $\cap G(f)=\emptyset$.

Lemma 4.7. A graph $G(f)$ of a function $f:(X, \tau) \rightarrow(Y, \sigma)$ is $(e, \theta)$ closed in $X \times Y$ if and only if for each $(x, y) \in(X \times Y) \backslash G(f)$, there exist a $\theta$-open set $U$ of $X$ containing $x$ and an e-open set $V$ of $Y$ containing $y$ such that $f(U) \cap V=\emptyset$.

Proof. It is an immediate consequence of Definition 8.

Theorem 4.8. If $f:(X, \tau) \rightarrow(Y, \sigma)$ is strongly faint e-continuous function and $(Y, \sigma)$ is $e-T_{2}$, then $G(f)$ is $(e, \theta)$-closed.

Proof. Let $(x, y) \in(X \times Y) \backslash G(f)$, then $f(x) \neq y$. Since $Y$ is $e-T_{2}$, there exist disjoint $e$-open sets $V$ and $W$ in $Y$ such that $f(x) \in V$ and $y \in W$. Since $f$ is strongly faint $e$-continuous, $f^{-1}(V)$ is $\theta$-open in $X$ containing $x$ . Take $U=f^{-1}(V)$. We have $f(U) \subset V$. Therefore, we obtain $f(U) \cap W$ $=\emptyset$. This shows that $G(f)$ is $(e, \theta)$-closed.

Theorem 4.9. Let $f:(X, \tau) \rightarrow(Y, \sigma)$ has an $(e, \theta)$-closed graph $G(f)$. If $f$ is a strongly faint e-continuous injection, then $(X, \tau)$ is $\theta-T_{2}$. 
Proof. Let $x$ and $y$ be any two distinct points of $X$. Then since $f$ is injective, we have $f(x) \neq f(y)$. Then, we have $(x, f(y)) \in(X \times Y) \backslash G(f)$. By Lemma 4.7, there exist a $\theta$-open set $U$ of $X$ and an $e$-open set $V$ of $Y$ such that $(x, f(y)) \in U \times V$ and $f(U) \cap V=\emptyset$. Hence $U \cap f^{-1}(V)=\emptyset$ and $y \notin U$. Since $f$ is strongly faint $e$-continuous, there exists a $\theta$-open set $W$ of $X$ containing $y$ such that $f(W) \subset V$. Therefore, we have $f(U) \cap f(W)$ $=\emptyset$. Since $f$ is injective, we obtain $U \cap W=\emptyset$. This implies that $(X, \tau)$ is $\theta-T_{2}$.

Definition 9. A topological space $X$ is said to be e-Alexandroff if every finite intersection of e-open sets is e-open.

Theorem 4.10. Let $(Y, \sigma)$ be e-Alexandroff. If $f:(X, \tau) \rightarrow(Y, \sigma)$ has the $(e, \theta)$-closed graph, then $f(K)$ is e-closed in $(Y, \sigma)$ for each subset $K$ which is $\theta$-compact relative to $X$.

Proof. Suppose that $y \notin f(K)$. Then $(x, y) \notin G(f)$ for each $x \in K$. Since $G(f)$ is $(e, \theta)$-closed, there exist a $\theta$-open $U_{x}$ set of $X$ containing $x$ and $e$-open $V_{x}$ set of $Y$ containing $y$ such that $f\left(U_{x}\right) \cap V_{x}=\emptyset$. by Lemma 4.7. The family $\left\{U_{x}: x \in K\right\}$ is a cover of $K$ by $\theta$-open sets. Since $K$ is $\theta$-compact relative to $(X, \tau)$, there exists a finite subset $K_{0}$ of $K$ such that $K \subset \bigcup\left\{U_{x}: x \in K_{0}\right\}$. Set $V=\bigcap\left\{V_{x}: x \in K_{0}\right\}$. Then $V$ is a $e$-open set in $Y$ containing $y$. Therefore, we have $f(K) \cap V \subset\left[\bigcup_{x \in K_{0}} f\left(U_{x}\right)\right] \cap V$ $\subset \bigcup_{x \in K_{0}}\left[f\left(U_{x}\right) \cap V\right]=\emptyset$. It follows that $y \notin e-c l(f(K))$. Therefore, $f(K)$ is $e$-closed in $(Y, \sigma)$.

Corollary 4.11. Let $(Y, \sigma)$ be e-Alexandroff. If $f:(X, \tau) \rightarrow(Y, \sigma)$ is strongly faint $e$-continuous and $(Y, \sigma)$ is $e-T_{2}$, then $f(K)$ is e-closed in $(Y, \sigma)$ for each subset $K$ which is $\theta$-compact relative to $(X, \tau)$.

Proof. The proof follows from Theorems 4.8 and 4.10 .

Acknowledgement. The authors are very grateful to the referee for your helpful suggestions.

\section{References}

[1] M. E. Abd El-Monsef, S. N. Ei-Deeb and R. A. Mahmoud, $\beta$-open sets and $\beta$-continuous mappings, Bull. Fac. Assiut Univ. 12, pp. 7790, (1983). 
[2] M. Caldas, On fantly e-continuous functions. Submitted.

[3] M. Caldas, S. Jafari and T. Noiri, Some separation axioms via modified $\theta$-open, Bull. of the Iranian Math. Soc., 29(2), pp. 1-12, (2003).

[4] E. Ekici, On $e$-open sets, $D P^{*}$-sets and $D P C^{*}$-sets and decompositions of continuity. The Arabian J. for Sci. and Eng., 33 (2A), pp. 269-282, (2008).

[5] E. Ekici, On $a$-open sets, $A^{*}$-sets and Decompositions of continuity and super continuity, Annales Univ. Sci. Budapest, 51, pp. 39-51, (2008).

[6] E. Ekici, New forms of contra continuity, Carpathian J. Math., 24 (1), pp. $37-45,(2008)$.

[7] E. Ekici, On $e^{*}$-open sets and $(D, S)^{*}$-sets, Math. Moravica, Vol. 13(1), pp. 29-36, (2009).

[8] E. Ekici, Some generalizations of almost contra-super-continuity, Filomat, 21 (2), pp. 31-44, (2007).

[9] E. Ekici, A note on $a$-open sets and $e^{*}$-sets, Filomat, 21 (1), pp. 89-96, (2008).

[10] A.A. El-Atik, Study of some types of mappings on topological spaces, Bull.Masters. Thesis, Faculty of Science, Tanta University, Egypt, (1997).

[11] S. Jafari, Some properties of quasi $\theta$-continuous functions, Far East J. Math. Soc., 6, pp. 689-696, (1998).

[12] N. Levine, Semi-open sets and semi-continuity in topological spaces, Amer. Math. Monthly, 68, pp. 44-46, (1961).

[13] N. Levine, Generalized closed sets in topology, Rend. Circ. Math. Palermo, 19, pp. 89-96, (1970).

[14] P. E. Long and L. L. Herrington, The $T_{\theta}$-topology and faintly continuous functions, Kyungpook Math. J., 22, pp. 7-14, (1982).

[15] A. S. Mashhour, M. E. Abd El-Monsef and S. N. El-Deeb, On precontinuous and weak precontinuous mappings, Proc. Math. Phys. Soc. Egypt, 53, pp. 47-53, (1982). 
[16] A. A. Nasef, Recent progress in the theory of faint continuity, Mathematical and Computer Modelling, 49, pp. 536-541, (2009).

[17] A. A. Nasef, strongly $\beta$-irresolute functions, J. Natur. Sci. Math., 36, pp. 199-206, (1996).

[18] A. A. Nasef and T. Noiri, Strong forms of faint continuity, Mem. Fac. Sci. Kochi Univ. Ser. A. Math, 19, pp. 21-28, (1998).

[19] O. Njåstad, On some classes of nearly open sets, Pacific J. Math., 15, pp. 961-970, (1965).

[20] T. Noiri, On $\delta$-continuous functions, J. Korean Math. Soc., 16, pp. 161-166, (1980).

[21] S. Raychaudhuri and M. N. Mukherjee, On $\delta$-almost continuity and $\delta$-preopen sets, Bull. Inst. Math. Acad. Sinica 21, pp. 357-366, (1993).

[22] S. Sinharoy and S. Bandyopadhyay, On $\theta$-completely regular and locally $\theta$-H-closed spaces, Bull. Cal. Math. Soc., 87, pp. 19-28, (1995).

[23] N. V. Veličko, $H$-closed topological spaces, Mat. Sb., 70 (1966), 98112; English transl., in Amer. Math. Soc. Transl., 78, pp. 103-118, (1968).

\section{Caldas}

Departamento de Matemática Aplicada, Universidade Federal Fluminense,

Rua Mário Santos Braga, s/n ${ }^{o}$ 24020-140, Niterói, RJ BRAZIL.

e-mail: gmamccs@vm.uff.br

and

\section{Saeid Jafari}

College of Vestsjaelland South,

Herrestraede 11,

4200 Slagelse,

Denmark

e-mail: jafari@stofanet.dk 